\title{
Reflets
}

Revue d'intervention sociale et communautaire

\section{Inégalités croissantes, écarts démesurés}

\section{Komi Hemedzo et Rachid Bagaoui}

Volume 24, numéro 1, printemps 2018

URI : https://id.erudit.org/iderudit/1051517ar

DOI : https://doi.org/10.7202/1051517ar

Aller au sommaire du numéro

Éditeur(s)

Reflets, Revue d'intervention sociale et communautaire

ISSN

1203-4576 (imprimé)

1712-8498 (numérique)

Découvrir la revue

Citer ce document

Hemedzo, K. \& Bagaoui, R. (2018). Inégalités croissantes, écarts démesurés. Reflets, 24(1), 10-15. https://doi.org/10.7202/1051517ar d'utilisation que vous pouvez consulter en ligne.

https://apropos.erudit.org/fr/usagers/politique-dutilisation/ 


\title{
Inégalités croissantes, écarts démesurés
}

\author{
Komi Hemedzo, Ph. D. \\ Professeur associé, \\ Département de sociologie, Université Laurentienne
}

\author{
Rachid Bagaoui, Ph. D. \\ Professeur agrégé, \\ Département de sociologie, Université Laurentienne
}

Ce numéro 1 du volume 24 de Reflets : revue d'intervention sociale et communautaire, du printemps 2018, est consacré aux diverses formes d'inégalités sociales en contexte minoritaire. En proposant cette thématique aux lectrices et lecteurs, le comité de rédaction de ce numéro a fait le pari de passer au crible de la recherche les données probantes et les revues scientifiques portant sur la question des inégalités. Au demeurant, l'objectif poursuivi est d'aboutir à de nouvelles connaissances sur la question et à une nouvelle compréhension de cette problématique, sujette aux changements à plusieurs niveaux. Ce sont ces mutations constantes qui font ainsi l'objet de recherches et d'analyses diverses et variées.

Les inégalités sociales en contexte minoritaire font l'objet de recherches récurrentes en sciences sociales dans la mesure où ces enjeux sont perceptibles dans toutes les sphères de la société. Si les chercheures et chercheurs se spécialisent par rapport à telles ou telles autres formes d'inégalités, c'est souvent par rapport aux inégalités de santé ou encore aux inégalités face au revenu. Ainsi, si l'on reconnait, par exemple, que les liens entre inégalités sociales et inégalités de santé sont imbriqués à telle enseigne que ces dernières sont " essentiellement le résultat, le produit final des autres inégalités sociales " (Aïach et Fassin, 2004, p. 2225), il en serait de même pour les autres types d'inégalités. Ce qui voudrait dire que les inégalités de revenu, les inégalités face à l'emploi sont à explorer sous leurs formes globales que constituent les inégalités sociales. Par ailleurs, les définitions sont nombreuses à propos du concept. La pertinence de cette thématique des inégalités réside dans le fait que les chercheures et chercheurs qui ont planché sur cette problématique n'ont pas couvert la diversité des aspects possibles des inégalités sociales. 
Or, il est impossible de l'aborder de façon exhaustive. Par exemple, Nicolas Herpin (1993) avait étudié les inégalités sociales sous différents angles, dont l'emploi et la ségrégation, la formation du couple et la famille, le seuil monétaire de la pauvreté et l'exclusion. Nicolas Duvoux (2017), quant à lui, dans Les inégalités sociales, souligne tout d'abord son caractère conventionnel avant d'aboutir à deux constats importants selon lesquels, d'une part, les pauvres sont de plus en plus pauvres en valeur absolue et, d'autre part, les inégalités sont produites par "l'assignation à une identité ", comme celle de la femme et de l'homme. Cette assignation est visible aussi sur le plan des identités linguistiques, des identités ethniques et raciales ou encore des identités de classe. D’ailleurs, tous les articles présentés dans ce numéro de Reflets touchent d'une manière ou d'une autre la question des identités sous l'angle des inégalités.

Dans un premier temps, les écrits sur les inégalités sociales sont nombreux, ce qui donne l'impression que ce champ soit assez exploré et qu'il faille abandonner toute quête. Dans un second temps, la société est en perpétuelle mutation à telle enseigne que de nouveaux problèmes sociaux surgissent sur le plan des inégalités. Certes, le milieu minoritaire n'est pas homogène, comme l'a bien noté Donald Dennie (1978), il y a 40 ans, dans un article intitulé De la diffculté d'être idéologue franco-ontarien. En s'appuyant sur l'exemple de la communauté franco-ontarienne, l'auteur estime qu'elle n'est pas homogène et qu'elle est traversée par les contradictions de classes, de sexe, etc. Si cette lecture a peut-être été valable il y a 40 ans, il faut reconnaître que le savoir en contexte minoritaire s'est beaucoup diversifié depuis, tenant compte de la différenciation inhérente à toute formation sociale. Le présent numéro s'inscrit dans cette mouvance.

En effet, il y a des inégalités non seulement entre la minorité et la majorité, mais aussi à l'intérieur de la minorité elle-même. C'est le cas des inégalités en contexte minoritaire exposées dans ce numéro à travers divers aspects, dont l'accès à la santé, l'accès au marché du travail, etc. Ainsi, ce numéro de la revue Reflets, loin de rompre cette habitude scientifique, apporte de nouveaux angles d'analyse aux questions des inégalités sociales. C'est un dépassement par un saut qualitatif en termes de prise en compte de l'émergence des aspects nouveaux des inégalités. D'ailleurs, Leslie Laczko (1998) pense que les évolutions notées sur ce sujet résident dans le fait que les inégalités sont diversement réparties sur le territoire canadien, d'où la nécessité pour les chercheures et chercheurs de faire preuve de perspicacité en termes d'approche méthodologique. Or, ces nouvelles approches s'ancrent dans le sillage de certains travaux comme ceux de Pierre Chauvin et Isabelle Parizot (2005), qui ont mis en corrélation inégalité sociale et territoire en concluant que les indicateurs de santé sont très défavorables et révélateurs dans les quartiers 
dits populaires. Ainsi, de manière factuelle, les habitants de quartiers populaires sont moins susceptibles d'avoir accès aux soins sous diverses formes. Chauvin et Parizot concluent ainsi qu'il y a aussi des disparités à l'intérieur d'un même groupe en ce qui concerne l'accès aux soins. Cette étude confirme l'approche axée sur le concept de capital social des francophones en situation minoritaire au Canada, chère à Louise Bouchard et Anne Gilbert (2005). Cette analyse est ancrée dans la tradition bourdieusienne de capital social et de reproduction sociale. En conclusion, les auteures ont concédé que le capital social n'est pas une fin en soi, mais elles le considèrent comme un concept important pouvant permettre de cerner les processus sociaux et les stratégies collectives dans l'analyse du développement des minorités francophones du Canada. On peut retenir essentiellement de cette analyse que Bouchard et Gilbert (2005) ont exposé trois niveaux de capital social, allant du niveau macro, où les réseaux francophones sont évoqués dans une perspective plus vaste avec une visée par rapport à leur insertion dans une collectivité, au niveau micro, où l'accent est mis sur l'individu dans ses interactions au sein de la société, et au niveau méso, où les réseaux sociaux sont intrinsèquement évoqués.

En somme, le présent numéro de Reflets approfondit de nombreuses dimensions des formes d'inégalités sociales. Les différents auteurs et auteures s'appuient sur plusieurs études récentes tournant autour de divers aspects de ce thème. Ce numéro se veut une tribune où toutes les recherches portant sur les inégalités sociales peuvent avoir un écho scientifique et, qui plus est, en milieu minoritaire. C'est une agora de savoirs portant sur la question des inégalités en pleine mutation. Le numéro présente des recherches novatrices et heuristiques issues des divers contextes géographiques au Canada, où certaines franges de la population sont en situation de minorité. Il propose un éventail d'analyses plus ou moins larges sur la thématique. Ainsi, les contributions des auteures et auteurs touchent différentes facettes de la thématique des inégalités, à savoir l'intégration à l'emploi des personnes présentant une déficience intellectuelle, le vieillissement au travail en contexte d'innovation, l'analyse intersectionnelle des défis et enjeux des inégalités sociales, puis le parcours d'intégration socioéconomique des nouvelles et nouveaux arrivants francophones à Ottawa. Ces problématiques sont traitées en fonction des différents contextes et milieux de vie minoritaires, l'Ontario et le Québec en particulier.

Le présent numéro comporte deux entrevues, la première ayant été réalisée auprès de David Macdonald, directeur du Centre canadien de politiques alternatives (CCPA), dont le mandat est de proposer une politique alternative au gouvernement en soumettant des budgets alternatifs. Cette entrevue porte sur le rôle et la politique du CCPA et a pour titre Inégalités et politiques alternatives. Après avoir fait la présentation de cette institution, 
David Macdonald explique les interventions du CCPA sur la question des inégalités. Depuis trente-huit ans, cette organisation travaille sur les questions des inégalités. Mais, durant les six dernières années, elle s'est concentrée sur le fossé qui se creuse de plus en plus entre riches et pauvres, alors que les premiers voient leurs revenus croître de façon exponentielle et les derniers en perdent considérablement. Il brosse aussi le portrait des inégalités au Canada en général et en Ontario en particulier. L'entretien se termine par la présentation d'une politique alternative en faveur de la lutte contre les inégalités.

La seconde entrevue a été effectuée auprès de Donald Dennie, professeur émérite de l'Université Laurentienne, dont les travaux portent sur les inégalités en milieu minoritaire francophone. L'entrevue s'intitule Néo-libéralisme, inégalités et minorité : le point de vue d'un sociologue. Elle retrace le parcours professionnel de Donald Dennie, grâce auquel il a découvert Karl Marx et, par ricochet, les classes sociales et les inégalités sociales. D’ailleurs, il tente de donner une définition du concept des inégalités en l'abordant sous les diverses formes qu'on rencontre dans la société, en l'occurrence la pauvreté, les inégalités culturelles, la situation minoritaire, etc. Selon lui, une minorité linguistique n'est pas forcément une minorité économique. Il fait aussi cas de ses engagements dans la communauté. Durant cet entretien, il parle des transformations sociales qui ont eu lieu à Sudbury, tout particulièrement avec la disparition de l'aristocratie ouvrière causée par la transformation du tissu économique axé sur les services et l'administration. Donald Dennie constate la disparition de la lutte syndicale. Lentretien se termine par sa déclaration d'amour à Erving Goffman, puisqu'il lui a permis de découvrir ce qu'il nomme la représentation de soi.

La section Le dossier offre quatre articles. Le premier est intitulé L'intégration à l'emploi des personnes présentant une déficience intellectuelle (DI) en situation linguistique minoritaire: état des lieux et pratiques prometteuses. Ses auteures, soit Lynne A. Potvin, Natasha Plourde, Linda Cardinal et Virginie Cobigo, se penchent sur la situation particulière des francophones présentant une DI. Dans un premier temps, elles présentent la recension des écrits scientifiques sur la formation professionnelle et l'intégration à un emploi des personnes présentant une DI. Ensuite, elles font le lien avec l'inclusion sociale et, surtout, avec la question des inégalités sociales. Les résultats de recherche et leur conclusion montrent que les francophones ayant une DI sont en manque de visibilité.

Le deuxième article a pour titre Vieillir au travail en contexte d'innovation : au-delà de la stigmatisation pour des pistes d'intégration. Marie-Michèle Lord et Pierre-Yves Therriault en sont les signataires. L'article aborde la question des problèmes de vieillissement, dont la marginalisation que peuvent vivre certaines personnes aînées sur le marché de 
l'emploi. Outre les aspects théorique et qualitatif de leur recherche, Lord et Therriault montrent que le vieillissement peut être un facteur d'analyse et d'expertise au travail. Leur démonstration aboutit au constat selon lequel vieillir au travail dans un contexte de nouveauté soulève un paradoxe, du fait que la « logique de création et de développement de nouvelles habiletés » est contradictoire à la " logique de compensation de certaines difficultés et de recherche de stabilité pour le faire».

Quant au troisième article, il est écrit par Nsimire Namululi, Rachid Bagaoui et Komi Hemedzo. Son titre est Analyse intersectionnelle des défis et enjeux des inégalités sociales sur le marché du travail : l'expérience de vingt femmes congolaises résidant au Québec et en Ontario. Comme l'indique son titre, l'article mobilise les différents marqueurs identitaires, dont la couleur de la peau, le sexe, l'origine ethnique, la taille, la langue et la religion, pour pouvoir expliquer les difficultés rencontrées par vingt femmes congolaises immigrantes vivant plus précisément en Ontario et au Québec. Après avoir décrit les divers obstacles vécus dans l'insertion professionnelle de ces femmes issues de l'immigration, l'article se termine sur la notion "il faut se battre ", qui serait un facteur de motivation et de résilience pour ces femmes face aux diverses adversités qu'elles rencontrent sur le marché de l'emploi.

Enfin, dans l'article intitulé Le parcours d'intégration socioéconomique des nouveaux arrivants francophones à Ottawa : quels effets sur la santé?, Jean-Marie Kouyé et Marguerite Soulière abordent les difficultés socioéconomiques majeures auxquelles sont confrontés les immigrantes et immigrants francophones à Ottawa. Kouyé et Soulière évoquent plusieurs contraintes, dont la barrière de langue (la non-maîtrise de l'anglais), les problèmes de logement, les problèmes de santé et surtout la non-reconnaissance de leurs diplômes. L'article se termine en relevant non seulement l'apport de la méthode participante utilisée, mais aussi la nécessité pour les pouvoirs publics de mettre en place une véritable politique d'intégration sociale et économique pour les nouveaux arrivants francophones.

Sous la rubrique Des pratiques à notre image, deux articles sont publiés. Le premier, écrit par Nathalie Morgan, s'intitule L'Alliance des femmes de la francophonie canadienne (AFFC) : pour et par les femmes francophones vivant en situation minoritaire au Canada. Morgan dresse l'historique de l'AFFC, un organisme fondé il y a plus de 100 ans, et en retrace l'évolution depuis sa création jusqu'à aujourd'hui, tout en présentant ses objectifs actuels. Le deuxième article, rédigé par Valérie Fortier, Michelle Tabor et Stéphanie Garneau, a pour titre L'insertion professionnelle des migrants francophones à Ottawa : un processus à double face. Les auteures s'appuient sur le Centre des services communautaires de Vanier (CSCV) à Ottawa pour mettre en lumière les défis auxquels font face les travailleuses et travailleurs migrants qualifiés à leur arrivée à Ottawa. Elles concluent 
que l'intégration de ces personnes incombe à la fois à la communauté d'accueil et aux personnes migrantes.

Le comité de rédaction de ce numéro de Reflets espère que les lectrices et lecteurs prendront plaisir à explorer les différentes rubriques et leurs contenus.

Bonne lecture!

\section{Bibliographie}

AÏACH, Pierre, et Didier FASSIN (2004). «L'origine et les fondements des inégalités sociales de santé », La Revue du Praticien, Vol. 54, p. 2221-2227.

BOUCHARD, Louise, et Anne GILBERT (2005). « Capital social et minorités francophones au Canada », Francophonies d'Amérique, № 20, p. 147-159.

CHAUVIN, Pierre, et Isabelle PARIZOT (2009). " Les inégalités sociales et territoriales de santé dans l'agglomération parisienne. Une analyse de la cohorte Sirs (2005) », Les cahiers de l'ONZUS, Délégation interministérielle à la Ville, $105 \mathrm{p}$.

DENNIE, Donald (1978). « De la difficulté d'être idéologue franco-ontarien », Revue du NouvelOntario, No 1, p. 69-90.

DUVOUX, Nicolas (2017). Les inégalités sociales, Paris, PUF, Collection Que sais-je?

HERPIN, Nicolas (1993). «L'urban underclass chez les sociologues américains : exclusion sociale et pauvreté », Revue française de sociologie, Vol. 34, №3, p. 421-439.

LACZKO, Leslie (1998). " Inégalités et État-providence : le Québec, le Canada et le Monde », Recherches sociographiques, Vol. 39, Nos 2 et 3, p. 317-340. 